\title{
Neutrophil-to-Lymphocyte Ratio Predicts Overall Survival of Advanced Non-Small Cell Lung Cancer Harboring Mutant Epidermal Growth Factor Receptor
}

\author{
Seigo Minami ${ }^{a}$,, Yoshitaka Ogata ${ }^{a}$, Shouichi Ihara ${ }^{a}$, Suguru Yamamoto ${ }^{\text {a }}$, Kiyoshi Komuta ${ }^{\mathrm{a}}$
}

\begin{abstract}
Background: Neutrophil-to-lymphocyte ratio (NLR) and lymphocyte-to-monocyte ratio (LMR) have been demonstrated to be prognostic biomarkers in various cancers, including non-small cell lung cancer (NSCLC). However, little has been known about these two ratios for a specific population of NSCLC harboring active epidermal growth factor receptor (EGFR) mutation.
\end{abstract}

Methods: We retrospectively reviewed electrical medical records of 152 patients who met the following criteria: NSCLC harboring mutant EGFR, EGFR-tyrosine kinase inhibitor (EGFR-TKI) monotherapy initiated between October 2007 and February 2017 at our hospital, stage III-IV or post-surgical recurrence. We compared overall survival (OS) and progression-free survival (PFS) between dichotomized groups by the optimal cut-off points of the two biomarkers. Univariate and multivariate Cox hazard analyses also searched for prognostic factors of survival time.

Results: OSs of NLR $<2.11$ (median 38.6 vs. 24.1 months, $\mathrm{P}<$ 0.01 ) and $L M R \geq 5.09$ (median 39.4 vs. 26.4 months, $\mathrm{P}<0.01$ ) were significantly longer than those of NLR $\geq 2.11$ and $\mathrm{LMR}<5.09$. Multivariate analyses found lower NLR (hazard ratio (HR) 1.07, 95\% CI: $1.01-1.14 ; \mathrm{P}=0.03$ ) as an independent prognostic factor for longer OS, in addition to Eastern Cooperative Oncology Group performance status 0 - 1, first-line EGFR-TKI, higher serum sodium concentration and lower lactate dehydrogenase. However, LMR was not detected as a significant prognostic factor for OS. None of these two biomarkers was selected as an independent prognostic factor for PFS.

Conclusions: This study demonstrated that elevated NLR is an independent prognostic factor for poor survival of patients with EGFR mutant NSCLC. NLR is a useful and simple biomarker for these patients.

Manuscript submitted November 28, 2017, accepted December 12, 2017

aDepartment of Respiratory Medicine, Osaka Police Hospital, 10-31 Kitayama-cho, Tennoji-ku, Osaka 543-0035, Japan

${ }^{b}$ Corresponding Author: Seigo Minami, Department of Respiratory Medicine, Osaka Police Hospital, 10-31 Kitayama-cho, Tennoji-ku, Osaka-City, Osaka 543-0035, Japan. Email: seigominami@oph.gr.jp

doi: https://doi.org/10.14740/wjon1069w
Keywords: Neutrophil-to-lymphocyte ratio; Lymphocyte-to-monocyte ratio; Non-small cell lung cancer; Epidermal growth factor receptor mutation; Epidermal growth factor receptor tyrosine kinase inhibitors; Overall survival; Progression-free survival; Retrospective analysis

\section{Introduction}

Epidermal growth factor receptor (EGFR) mutation status divides non-small cell lung cancer (NSCLC) into two genetic subsets, NSCLC with positive EGFR mutation and NSCLC with wild-type EGFR. For the former subset, EGFR tyrosine kinase inhibitors (EGFR-TKIs) provide better efficacy and longer survival than conventional cytotoxic chemotherapy. Thus, exclusively for these selected patients, EGFR-TKIs are prioritized over conventional chemotherapy. However, these drugs are not always effective for them. Overall response rate ranged $60-80 \%$. Many patients experienced tumor progression 9 - 13 months after initiation of EGFR-TKIs [1-6].

Patient's systemic inflammatory response (SIR) plays an adverse role in development and prognosis of cancers [7-9]. Neutrophils, lymphocytes and monocytes are important for the cancer-induced SIR $[10,11]$. These three types of circulating cells represent the patients' SIR. Their peripheral absolute counts can predict prognoses of various malignancies, including NSCLC. According to a Japanese analysis of 388 chemonaive NSCLC with stage IIIB or IV, pretreatment elevated neutrophil count was significantly associated with shorter overall survival (OS) and progression-free survival (PFS). However, this study failed to show any association between prognosis and lymphocyte or monocyte count [12]. On the other hand, another Japanese multivariate analysis of 237 node-negative NCSLC demonstrated that a preoperative peripheral lymphocyte count was an independent prognostic factor for OS rate, but the neutrophil count was not [13]. A Chinese multivariate analysis of 142 patients with NSCLC who had underwent lobectomy, lymph node dissection and adjuvant chemotherapy also detected preoperative lymphocyte count, but not neutrophil count, as an independent prognostic factor of disease-free survival [14]. These two studies considered that peripheral lymphocyte count was associated with vascular or lymphatic invasion $[13,14]$. Regarding peripheral monocyte count, in a Chinese analysis of 370 patients who had received platinum-based doublet for pre- 
Table 1. Comparisons of Backgrounds and Laboratory Data Between Two Groups Divided According to Neutrophil-to-Lymphocyte and Lymphocyte-to-Monocyte Ratios

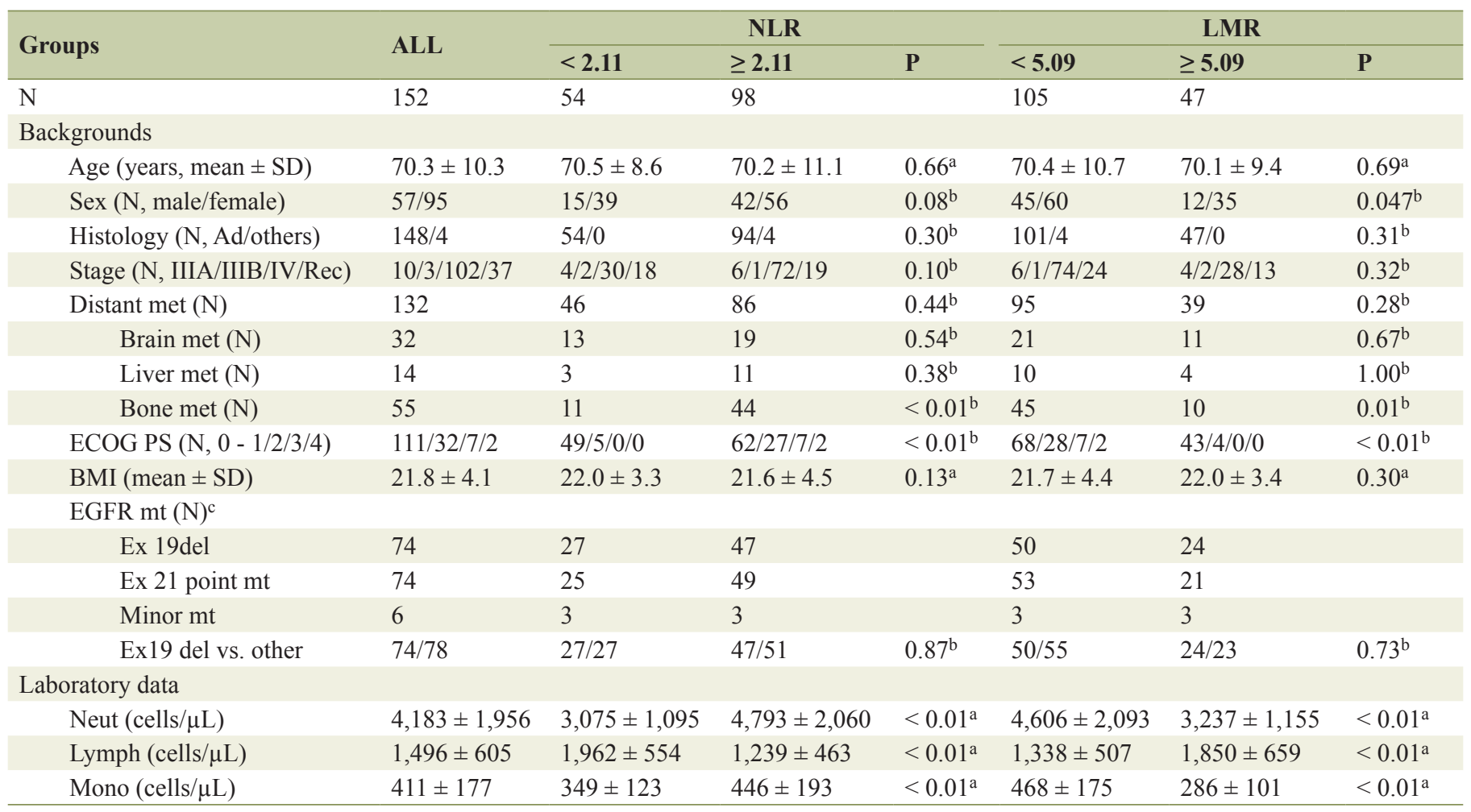

aMann-Whitney test. bFisher's extract test. cDouble mutations. Exon 19del + Exon 21 L858R and Exon 21 L858R + Exon 18 G719S in each one case. Ad: adenocarcinoma; BMI: body mass index; del: deletion; Cl: confidence interval; ECOG PS: Eastern Cooperative Oncology Group performance status; LMR: Iymphocyte-to-monocyte ratio; Lymph: lymphocyte; met: metastasis; Mono: monocyte; mt: mutation; Neut: neutrophil; NLR: neutrophilto-lymphocyte ratio; Rec: post-surgical recurrence; SD: standard deviation.

viously untreated metastatic NSCLC, monocyte count was an independent prognostic factor for PFS and OS [15].

Recently, there has been increasing evidences of the prognostic value of combined scoring systems based on the SIR. Especially, neutrophil-to-lymphocyte ratio (NLR) and lymphocyte-to-monocyte ratio (LMR) are easily calculated from leukocyte count and differentiation. Elevated NLR [16-18] and decreased LMR $[15,19]$ have been demonstrated to be associated with poor prognosis in various cancers, including advanced NSCLC. However, little has been known about these two ratios for a specific genetic subset of EGFR mutant NSCLC.

In this study, we evaluate the prognostic value of NLR and LMR in patients with EGFR mutant NSCLC.

\section{Methods}

This study was retrospective and single institutional. We collected the following patients: 1) patients who started EGFRTKIs monotherapy between October 2007 and February 2017, 2) histologically or cytologically proven NSCLC, and positive EGFR mutation status confirmed by the peptide nucleic acid-locked nucleic acid PCR clamp method by LSI Medience Cooperation (Tokyo, Japan) [20], 3) c-stage IIIA-B, IV or post-surgical recurrence, based on the 7 th TNM classifica- tion of lung cancer by the Union for International Cancer Control (UICC) [21], 4) patients who received blood test within 1 week before the initiation of EGFR-TKIs. In Japan, gefitinib, erlotinib and afatinib were approved for NSCLC in July 2002, October 2007 and January 2014, respectively. EGFR mutation tests became covered by insurance in June 2007. The definitions of overall response rate (RR), disease control rate (DCR), PFS and OS in this study followed those of our previous studies [22, 23]. When RR, DCR and PFS were calculated, sequential change from a TKI to another TKI due to adverse effects was arbitrarily defined as one regimen treatment in this study. When we calculated creatinine clearance (Ccr) by means of Cockcroft-Gault equation, we added $0.2 \mathrm{mg} / \mathrm{dL}$ on serum creatinine values determined by the enzymatic method [24]. Peripheral venous blood (approximately $2 \mathrm{~mL}$ ) was collected into a sterile ethylene-diamine-tetra-acetic acid (EDTA) tube. Hematological parameters were analyzed using hematology analyzers (Sysmex XE-2100 until 2012, and thereafter Sysmex XE-5000; Sysmex, Kobe, Japan). NLR and LMR were obtained by dividing pretreatment venous absolute circulating neutrophil count by lymphocyte count, and venous absolute circulating lymphocyte count by monocyte count, respectively. The data cut-off was September 1, 2017. The Osaka Police Hospital Ethics Committee approved this study (number 685), and waived the requirement for informed consent because de- 
Table 2. Treatment and Outcomes

\begin{tabular}{|c|c|c|c|c|c|c|c|}
\hline \multirow{2}{*}{ Group } & \multirow{2}{*}{ All } & \multicolumn{3}{|c|}{ NLR } & \multicolumn{3}{|c|}{ LMR } \\
\hline & & $<2.11$ & $\geq 2.11$ & $\mathbf{P}^{\mathbf{a}}$ & $<5.09$ & $\geq 5.09$ & $\mathbf{P}^{\mathbf{a}}$ \\
\hline $\mathrm{N}$ & 152 & 54 & 98 & & 105 & 47 & \\
\hline TKI choice (N, Gef/Erl/Afa) & $107 / 36 / 9$ & $43 / 10 / 1$ & $64 / 26 / 8$ & 0.12 & $72 / 27 / 6$ & $35 / 9 / 3$ & 0.68 \\
\hline Pre TKI treatment $(\mathrm{N})$ & 49 & 18 & 31 & 0.86 & 34 & 10 & 0.18 \\
\hline Platinum-based & 39 & 14 & 25 & 1.00 & 30 & 7 & 0.10 \\
\hline Cytotoxic drugs & 62 & 26 & 36 & 0.23 & 40 & 22 & 0.37 \\
\hline Platinum-based & 27 & 10 & 17 & 1.00 & 18 & 13 & 0.19 \\
\hline Osimertinib & 14 & 5 & 9 & 1.00 & 9 & 5 & 0.76 \\
\hline Re-challenge of TKI & 62 & 27 & 35 & 0.12 & 42 & 22 & 0.48 \\
\hline Palliative radiotherapy $(\mathrm{N})$ & 57 & 18 & 39 & 0.49 & 38 & 19 & 0.72 \\
\hline Stable disease & 29 & 11 & 18 & & 21 & 8 & \\
\hline Progressive disease & 19 & 2 & 17 & & 18 & 1 & \\
\hline Not evaluated & 11 & 2 & 9 & & 9 & 2 & \\
\hline RR (\%) $(95 \% \mathrm{CI})$ & $61.2(53.0-69.0)$ & $72.2(58.4-83.5)$ & $55.1(44.7-65.2)$ & 0.055 & $54.3(44.3-64.0)$ & $76.6(62.0-87.7)$ & 0.01 \\
\hline DCR $(\%)(95 \% \mathrm{CI})$ & $80.3(73.0-86.3)$ & $92.6(82.1-97.9)$ & $73.5(63.6-81.9)$ & $<0.01$ & $74.3(64.8-82.3)$ & $93.6(82.5-98.7)$ & $<0.01$ \\
\hline
\end{tabular}

aFisher's extract test. Afa: afatinib; Cl: confidence interval; DCR: disease control rate; Erl: erlotinib; Gef: gefitinib; LMR: Iymphocyte-to-monocyte ratio; NLR: neutrophil-to-lymphocyte ratio; RR: response rate; TKI: tyrosine kinase inhibitor; TRT: thoracic radiotherapy.

identified data were retrospectively collected.

We described the continuous, categorical and survival variables as the mean \pm standard deviation (SD), frequency, median (95\% confidential intervals (CIs)), respectively. We used Fisher's exact and Mann-Whitney tests for comparison of the relative frequencies and continuous variables that were not normally distributed, respectively. Using receiver operating characteristic (ROC) curve and Youden index, we selected the optimal cut-off values of NLR and LMR, and then divided our patients into two groups. We arbitrary defined complete and partial responses of EGFR-TKIs as the outcome variables of ROC analysis. The results of ROC analyses were expressed as sensitivity, specificity, area under the curve (AUC) with $95 \%$ CI. We used Kaplan-Meier method to evaluate PFS and OS, and compared two groups by log-rank test.

To investigate independent prognostic factors, we used Cox proportional hazard analyses. After the univariate analysis, only variables with P-value $<0.1$ were used in the multivariate analysis. The results were described as hazard ratios (HR) and 95\% CI. P-value $<0.05$ was defined as statistically significant. All statistical analyses were performed with EZR (Saitama Medical Center, Jichi Medical University, Saitama, Japan), which is a graphical user interface for R (The R Foundation for Statistical Computing, Vienna, Austria) [25].

\section{Results}

We collected 152 patients for this study. At the time of data-cut off, 85 patients were dead, 39 still alive and 28 lost to followup. As for the first TKI treatment, 15 patients still continued TKI, while the other 137 discontinued TKI because of PD in 84 , adverse effects in 20, deteriorated comorbidity or general condition in 17, change regimens from EGFR-TKI to cytotoxic drugs before PD in 5, patients' refusal in 3 , transfer to other hospitals in 6 and unidentified death in 2 . Eleven patients changed TKIs mainly due to adverse effects. As the optimal cut-off points of NLR and LMR, ROC curves defined 2.11 (sensitivity 0.75 , specificity 0.42 , AUC 0.54 , 95\% CI 0.44 0.64 ) and 5.09 (sensitivity 0.83 , specificity 0.39 , AUC 0.60, $95 \%$ CI $0.51-0.69)$, respectively.

High NLR group (NLR $\geq 2.11$ ) included more patients with bone metastases ( $45 \%$ vs. $20 \%, \mathrm{P}<0.01)$ and severe Eastern Cooperative Oncology Group performance status (ECOG PS) $(\mathrm{P}<0.01)$ than low NLR group (NLR $<2.11)$. High LMR group (LMR $\geq 5.09)$ was characteristics of more female $(74 \%$ vs. $57 \%, \mathrm{P}=0.047)$, less frequent bone metastases $(21 \%$ vs. $43 \%, \mathrm{P}=0.01)$, better PS $(\mathrm{P}<0.01)$ than low LMR group $($ LMR < 5.09) (Table 1). Patients with low NLR were more likely to receive further line chemotherapy $(76 \%$ vs. $58 \%, \mathrm{P}=$ $0.03)$. RR were significantly higher in high LMR group than in low LMR group (76.6\% vs. 54.3\%, $\mathrm{P}=0.01)$. DCRs were significantly higher in low NLR $(92.6 \%$ vs. $73.5 \%, \mathrm{P}<0.01)$ and high LMR $(93.6 \%$ vs. $74.3 \%, \mathrm{P}<0.01)$ groups than in high NLR and low LMR groups (Table 2). OS of low NLR (OS, 38.6 vs. 24.1 months in median, $\mathrm{P}<0.01$ ) and high LMR (OS, 39.4 vs. 26.4 months, $\mathrm{P}<0.01$ ) groups were significantly longer than those of high NLR and low LMR groups (Fig. 1). 
a Overall Survival according to NLR

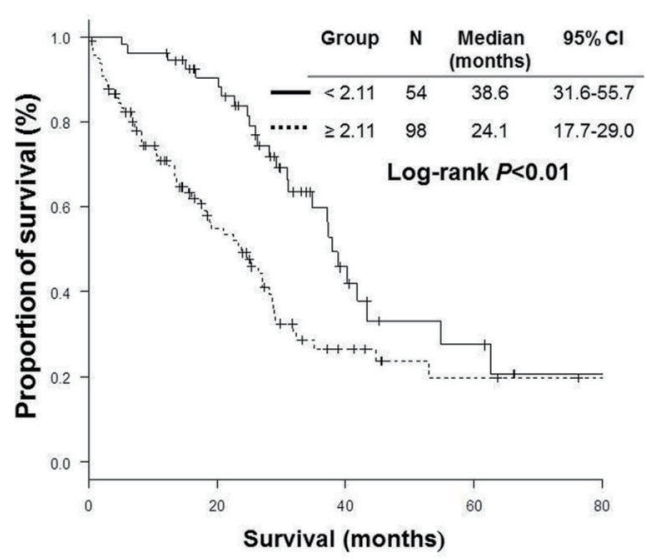

b Overall Survival according to LMR

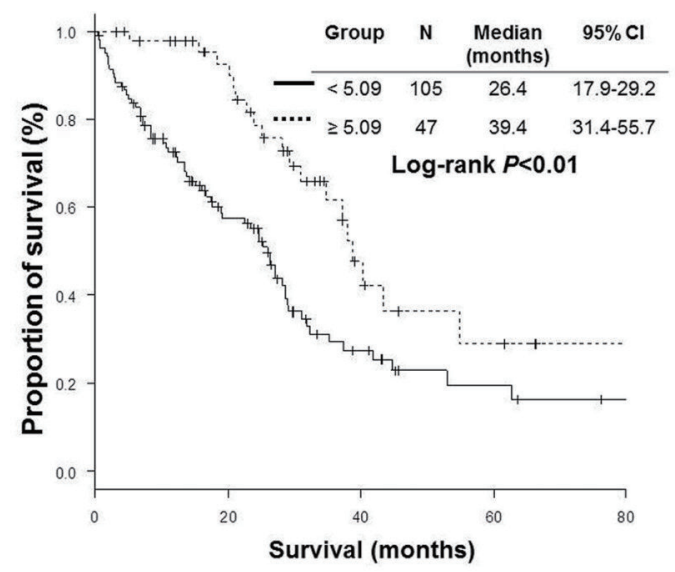

Figure 1. Kaplan-Meier curves of overall survival. (a) Neutrophil-to-lymphocyte ratio (NLR). (b) Lymphocyte-to-monocyte ratio (LMR).

However, PFS of low NLR (PFS, 15.9 vs. 10.1 months in median, $P=0.04$ ) group was significantly longer than those of high NLR group, while there was no statistically significant difference in PFS according to LMR (Fig. 2).

Univariate analyses detected ECOG PS 0 - 1, higher Ccr, higher sodium concentration, lower lactate dehydrogenase (LDH), lower C-reactive protein (CRP), lower NLR and higher LMR as prognostic factors for longer OS; no distant metastasis, ECOG PS 0 - 1, body mass index (BMI) $\geq 18.5$, EGFRTKI introduction in first-line setting, treatment with erlotinib or afatinib, higher Ccr, higher sodium concentration, lower LDH, lower CRP, lower NLR and higher LMR as predictive factors for longer PFS (Table 3). The subsequent multivariate analyses found lower NLR (HR 1.07, 95\%CI: 1.01 - 1.14; P $=0.03$ ) as an independent prognostic factor for longer OS, in addition to ECOG PS 0 - 1, first-line introduction of EGFRTKI, higher sodium concentration and lower LDH. However, LMR was not detected as a significant prognostic factor for OS (Table 4). Neither NLR nor LMR was selected as a significant prognostic factor for PFS (Table 5).

\section{Discussion}

This study was a comprehensive evaluation of two biomarkers and prognosis in patients with EGFR mutant NSCLC treated

\section{b PFS according to LMR}
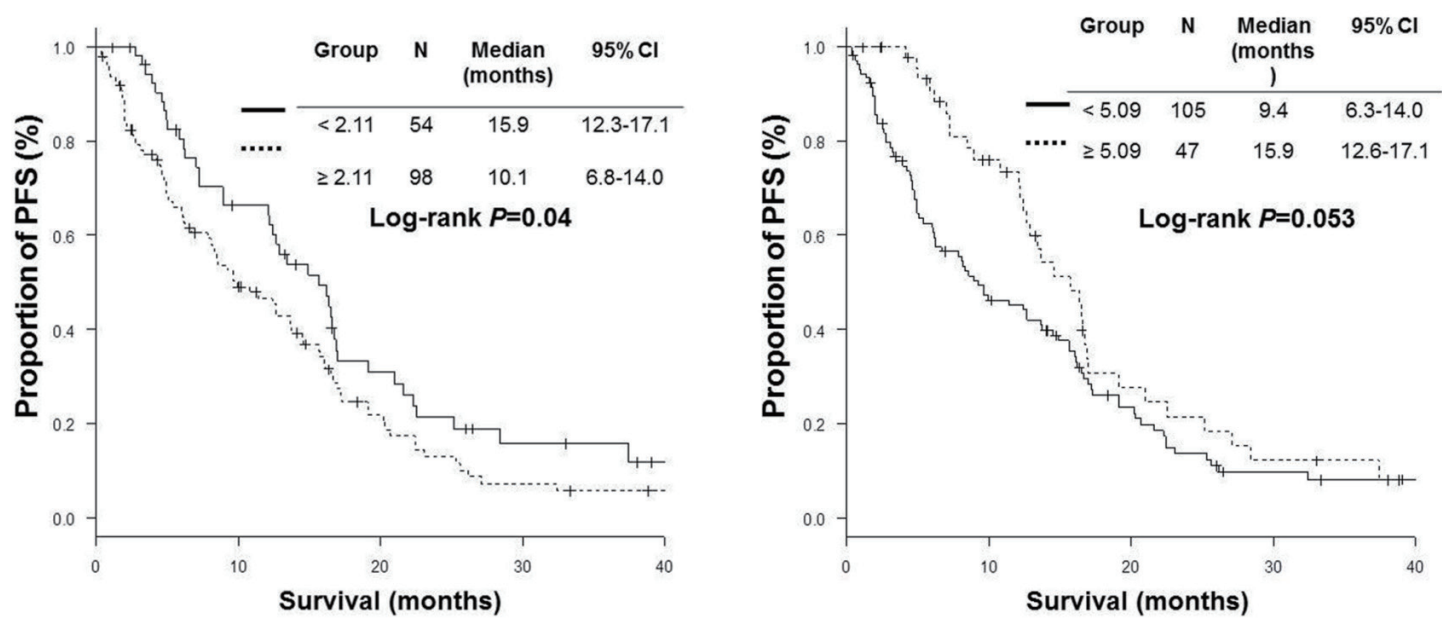

Figure 2. Kaplan-Meier curves of progression-free survival (PFS). (a) Neutrophil-to-lymphocyte ratio (NLR). (b) Lymphocyte-tomonocyte ratio (LMR). 
Table 3. Univariate Cox Hazard Analyses of Factors Associated With Overall Survival and Progression-Free Survival

\begin{tabular}{|c|c|c|c|c|c|c|}
\hline & & OS & & & PFS & \\
\hline & HR & $95 \%$ CI & $\mathbf{P}$ & HR & $95 \%$ CI & $\mathbf{P}$ \\
\hline Sex (female vs. male) & 1.33 & $0.86-2.07$ & 0.19 & 1.17 & $0.81-1.68$ & 0.41 \\
\hline ECOG PS (0 - 1 vs. 2 - 4) & 4.08 & $2.56-6.50$ & $<0.01$ & 1.98 & $1.33-2.93$ & $<0.01$ \\
\hline BMI $(\geq 18.5$ vs. $<18.5)$ & 1.58 & $0.95-2.64$ & 0.08 & 1.60 & $1.04-2.47$ & 0.03 \\
\hline EGFR-TKI (Gef vs. Erl or Afa) & 0.84 & $0.50-1.41$ & 0.51 & 0.61 & $0.40-0.92$ & 0.02 \\
\hline Hemoglobin $(\mathrm{g} / \mathrm{dL})$ & 0.89 & $0.77-1.02$ & 0.09 & 0.94 & $0.85-1.05$ & 0.29 \\
\hline $\operatorname{Ccr}(\mathrm{mL} / \mathrm{min})(/ 10)$ & 0.86 & $0.74-0.998$ & 0.047 & 0.88 & $0.78-0.99$ & 0.04 \\
\hline Sodium $(\mathrm{mEq} / \mathrm{L})(/ 10)$ & 0.19 & $0.10-0.39$ & $<0.01$ & 0.34 & $0.19-0.62$ & $<0.01$ \\
\hline LDH (IU/L) (/100) & 1.36 & $1.19-1.54$ & $<0.01$ & 1.24 & $1.11-1.39$ & $<0.01$ \\
\hline
\end{tabular}

Afa: afatinib; BMI: body mass index; Ccr: creatinine clearance; Cl: confidence interval; CRP: C-reactive protein; del: deletion; ECOG PS: Eastern Cooperative Oncology Group performance status; EGFR-TKI: epidermal growth factor receptor- tyrosine kinase inhibitor; Erl: erlotinib; Gef: gefinitib; HR: hazard ratio; LDH: lactate dehydrogenase; LMR: lymphocyte-to-monocyte ratio; NLR: neutrophil-to-lymphocyte ratio; OS: overall survival; PFS: progression-free survival.

with EGFR-TKIs. To our knowledge, there were only four studies and one study that had investigated association of NLR [26-29] and LMR [30] with prognosis, respectively (Table 6).

NLR is an independent prognostic factor for OS of those selected population. Our survival comparisons and multivariate analyses demonstrated that higher NLR was associated with poorer OS, and were consistent with previous three studies [26-28]. On the other hand, despite of significantly longer $\mathrm{OS}$ in patients with $\mathrm{LMR} \geq 5.09$, our multivariate analysis failed to detect LMR as a significant prognostic factor for OS. This result was different from the previous larger-scaled study [30]. Our study scale might be insufficient to evaluate LMR.

Both NLR and LMR are still controversial as prognostic factors for PFS of EGFR-TKI treatment. In our survival comparisons, PFSs of patients with NLR $\geq 2.11$ and LMR $<5.09$ were significantly shorter than those of patients with NLR $<$ 2.11 and $L M R \geq 5.09$, respectively. However, our multivariate analyses did not detect NLR and LMR as independent prognostic factors for PFS. As an independent prognostic factor for shorter PFS, NLR $\geq 3.5$ [27, 28] and NLR $\geq 5$ [26] were demonstrated by previous studies. However, a Korean study failed to demonstrate NLR $\geq 3$ as a significant prognostic factor [29]. On the other hand, a Taiwan study found that high baseline LMR was an independent prognostic factor for PFS of first-line TKIs [30], which was contrary to ours. Between the Taiwan and our studies, there seemed to be some differences in

Table 4. Multivariate Cox Hazard Analyses of Factors Associated With Overall Survival

\begin{tabular}{|c|c|c|c|c|c|c|}
\hline & & NLR & & & LMI & \\
\hline & HR & $95 \%$ CI & $\mathbf{P}$ & HR & $95 \%$ CI & $\mathbf{P}$ \\
\hline Distant metastasis (no vs. yes) & 1.59 & $0.73-3.50$ & 0.25 & 1.44 & $0.64-3.24$ & 0.38 \\
\hline ECOG PS (0 - 1 vs. $2-4)$ & 3.46 & $2.03-5.89$ & $<0.01$ & 3.41 & $1.99-5.82$ & $<0.01$ \\
\hline EGFR-TKI line (first vs. second or later) & 1.71. & $1.07-2.73$ & 0.02 & 1.72 & $1.08-2.74$ & 0.02 \\
\hline Hemoglobin $(\mathrm{g} / \mathrm{dL})$ & 1.14 & $0.97-1.34$ & 0.11 & 1.15 & $0.98-1.36$ & 0.08 \\
\hline LDH (IU/L) $(/ 100)$ & 1.24 & $1.05-1.48$ & 0.01 & 1.27 & $1.07-1.50$ & $<0.01$ \\
\hline $\mathrm{CRP}(\mathrm{mg} / \mathrm{dL})$ & 1.01 & $0.90-1.14$ & 0.82 & 0.98 & $0.86-1.11$ & 0.74 \\
\hline NLR & 1.07 & $1.01-1.14$ & 0.03 & - & - & - \\
\hline LMR & - & - & - & 0.88 & $0.76-1.01$ & 0.07 \\
\hline
\end{tabular}

BMI: body mass index; Ccr: creatinine clearance: Cl: confidence interval; CRP: C-reactive protein; ECOG PS: Eastern Cooperative Oncology Group performance status; EGFR-TKI: epidermal growth factor receptor-tyrosine kinase inhibitors; HR: hazard ratio; LDH: lactate dehydrogenase; LMR: lymphocyte-to-monocyte ratio; NLR: neutrophil-to-lymphocyte ratio. 
Table 5. Multivariate Cox Hazard Analyses of Factors Associated With Progression-Free Survival

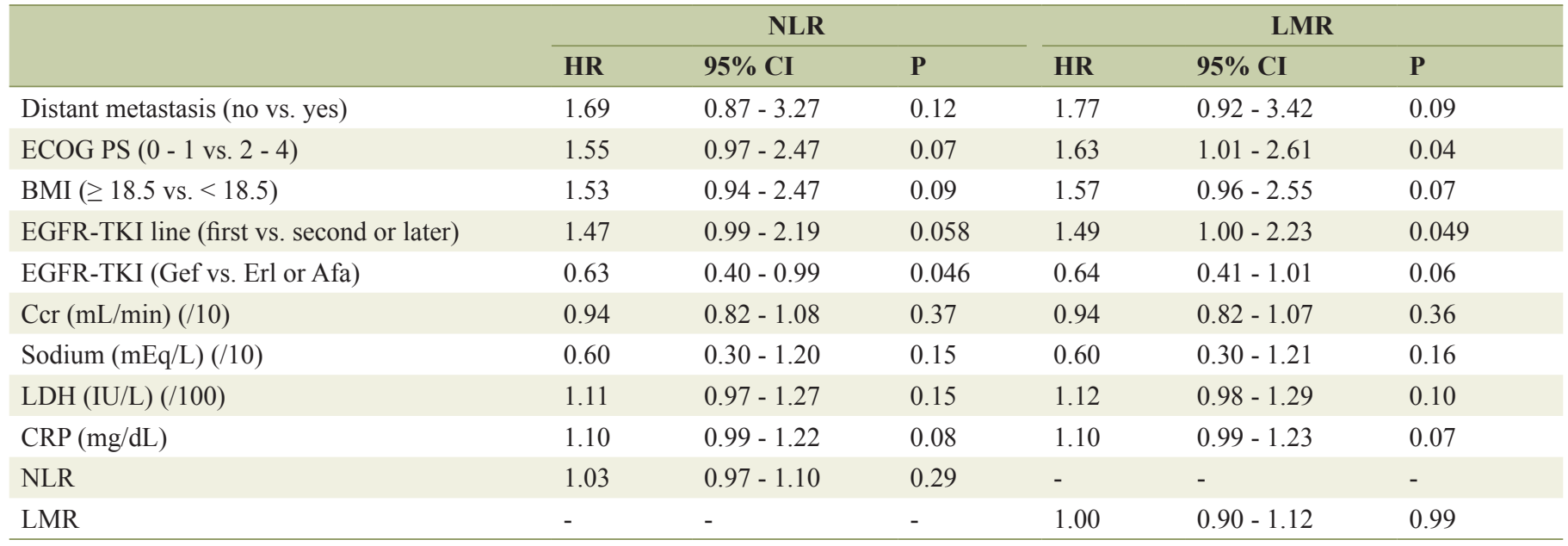

Afa: afatinib; ALP: alkaline phosphatase; BMI: body mass index; Ccr: creatinine clearance; Cl: confidence interval; CRP: C-reactive protein; ECOG PS: Eastern Cooperative Oncology Group performance status; EGFR-TKI: epidermal growth factor receptor-tyrosine kinase inhibitors; Erl: erlotinib; Gef: gefitinib; HR: hazard ratio; LDH: lactate dehydrogenase; LMR: lymphocyte-to-monocyte ratio; NLR: neutrophil-to-lymphocyte ratio.

age $(65.2 \pm 12.4$ vs. $70.3 \pm 10.3$ years $)$ and proportion of TKI in the first-line setting (100\% vs. 68.4\%). Furthermore, significant prognostic factors detected by multivariate analyses for PFS were different. Our study found ECOG PS (0 - 1 vs. 2 - 4) and TKI line setting (first vs. second or later line), while the Taiwan study detected LMR, the 1-month-to-basline ratio of LMR, EGFR mutation (common vs. uncommon) and distant metastases ( 0 - 2 vs. $>2)$. Thus, there is no established view on these two biomarkers as prognostic factors for PFS.

Our study included some limitations. First, we are afraid that our study was too small to find these two biomarkers as significant factors. Especially, our study underestimated LMR as a prognostic factor for OS. Second, our study might include case bias because of a single-centered and retrospective study. Multi-centered, prospective and larger-sized studies are ideal for such a specific cancer population.

\section{Conclusions}

This study demonstrated that elevated NLR is an independent prognostic factor for poor survival of patients with EGFR mutant NSCLC. NLR is a useful and simple biomarker for these patients, while LMR requires further studies as a significant

Table 6. Review of Previous Studies of Multivariate Analyses for Prediction of Survival Outcomes in Patients With EGFR Mutant NSCLC

\begin{tabular}{|c|c|c|c|c|c|c|c|c|c|}
\hline \multirow{3}{*}{$\begin{array}{l}\text { Author } \\
\text { (year) }\end{array}$} & \multirow{3}{*}{ Country } & \multirow{3}{*}{$\mathbf{N}$} & \multirow{3}{*}{ Treatment } & \multirow{3}{*}{ Variable } & \multicolumn{5}{|c|}{ Multivariate analyses } \\
\hline & & & & & \multirow{2}{*}{$\begin{array}{l}\text { Cut- } \\
\text { off }\end{array}$} & \multicolumn{2}{|c|}{ OS } & \multicolumn{2}{|l|}{ PFS } \\
\hline & & & & & & HR $(95 \%$ CI $)$ & $\mathbf{P}$ & HR $(95 \%$ CI) & $\mathbf{P}$ \\
\hline $\begin{array}{l}\text { Chen et al } \\
{[30](2015)}\end{array}$ & Taiwan & 253 & All first-line & LMR & 3.29 & $2.36(1.66-3.35)$ & $<0.001$ & $1.71(1.14-2.56)$ & 0.009 \\
\hline $\begin{array}{l}\text { Meriggi et al } \\
{[28](2017)}\end{array}$ & Italy & 63 & $\operatorname{Gef}(71 \%) \operatorname{Erl}(29 \%)$ & NLR & 3.5 & $2.699(1.187-6.137)$ & 0.018 & $2.275(1.257-4.116)$ & 0.007 \\
\hline $\begin{array}{l}\text { Ding et al } \\
{[26](2017)}\end{array}$ & Australia & 85 & First-line $(85 \%)$ & NLR & 5 & $0.43(0.19-0.94)$ & 0.04 & $0.40(0.18-0.87)$ & 0.02 \\
\hline
\end{tabular}

Cl: confidence interval; Erl: erlotinib; Gef: gefitinib; HR: hazard ratio; NA: not assessed; LMR: lymphocyte-to-monocyte ratio; NLR: neutrophil-tolymphocyte ratio; OS: overall survival; PFS: progression free survival. 
biomarker.

\section{Acknowledgments}

We are grateful to Sung-Ho Kim, Yoshimi Noda, Yuki Nakatani, Kanako Nishimatsu, Shouko Ikuta, Saori Ikebe and Hideyasu Okada at the Department of Respiratory Medicine, Osaka Police Hospital for their detailed medical records, diagnosis, treatment and care of their patients.

\section{Conflict of Interest}

The authors declare that they have no conflict of interest.

\section{Grant Support}

None.

\section{References}

1. Inoue A, Kobayashi K, Usui K, Maemondo M, Okinaga S, Mikami I, Ando M, et al. First-line gefitinib for patients with advanced non-small-cell lung cancer harboring epidermal growth factor receptor mutations without indication for chemotherapy. J Clin Oncol. 2009;27(9):13941400 .

2. Mitsudomi T, Morita S, Yatabe Y, Negoro S, Okamoto I, Tsurutani J, Seto T, et al. Gefitinib versus cisplatin plus docetaxel in patients with non-small-cell lung cancer harbouring mutations of the epidermal growth factor receptor (WJTOG3405): an open label, randomised phase 3 trial. Lancet Oncol. 2010;11(2):121-128.

3. Rosell R, Carcereny E, Gervais R, Vergnenegre A, Massuti B, Felip E, Palmero R, et al. Erlotinib versus standard chemotherapy as first-line treatment for European patients with advanced EGFR mutation-positive non-smallcell lung cancer (EURTAC): a multicentre, open-label, randomised phase 3 trial. Lancet Oncol. 2012;13(3):239246.

4. Sequist LV, Yang JC, Yamamoto N, O’Byrne K, Hirsh V, Mok T, Geater SL, et al. Phase III study of afatinib or cisplatin plus pemetrexed in patients with metastatic lung adenocarcinoma with EGFR mutations. J Clin Oncol. 2013;31(27):3327-3334.

5. Wu YL, Zhou C, Hu CP, Feng J, Lu S, Huang Y, Li W, et al. Afatinib versus cisplatin plus gemcitabine for first-line treatment of Asian patients with advanced non-small-cell lung cancer harbouring EGFR mutations (LUX-Lung 6): an open-label, randomised phase 3 trial. Lancet Oncol. 2014;15(2):213-222.

6. Zhou C, Wu YL, Chen G, Feng J, Liu XQ, Wang C, Zhang $\mathrm{S}$, et al. Erlotinib versus chemotherapy as first-line treatment for patients with advanced EGFR mutation-positive non-small-cell lung cancer (OPTIMAL, CTONG-0802): a multicentre, open-label, randomised, phase 3 study. Lancet Oncol. 2011;12(8):735-742.

7. Coussens LM, Werb Z. Inflammation and cancer. Nature. 2002;420(6917):860-867.

8. Mantovani A, Romero P, Palucka AK, Marincola FM. Tumour immunity: effector response to tumour and role of the microenvironment. Lancet. 2008;371(9614):771-783.

9. Proctor MJ, Morrison DS, Talwar D, Balmer SM, Fletcher CD, O'Reilly DS, Foulis AK, et al. A comparison of inflammation-based prognostic scores in patients with cancer. A Glasgow Inflammation Outcome Study. Eur J Cancer. 2011;47(17):2633-2641.

10. Schreiber RD, Old LJ, Smyth MJ. Cancer immunoediting: integrating immunity's roles in cancer suppression and promotion. Science. 2011;331(6024):1565-1570.

11. McMillan DC. Systemic inflammation, nutritional status and survival in patients with cancer. Curr Opin Clin Nutr Metab Care. 2009;12(3):223-226.

12. Teramukai S, Kitano T, Kishida Y, Kawahara M, Kubota K, Komuta K, Minato K, et al. Pretreatment neutrophil count as an independent prognostic factor in advanced non-small-cell lung cancer: an analysis of Japan Multinational Trial Organisation LC00-03. Eur J Cancer. 2009;45(11):1950-1958.

13. Kobayashi N, Usui S, Kikuchi S, Goto Y, Sakai M, Onizuka M, Sato Y. Preoperative lymphocyte count is an independent prognostic factor in node-negative non-small cell lung cancer. Lung Cancer. 2012;75(2):223-227.

14. Zhang J, Huang SH, Li H, Li Y, Chen XL, Zhang WQ, Chen HG, et al. Preoperative lymphocyte count is a favorable prognostic factor of disease-free survival in nonsmall-cell lung cancer. Med Oncol. 2013;30(1):352.

15. Lin GN, Peng JW, Xiao JJ, Liu DY, Xia ZJ. Prognostic impact of circulating monocytes and lymphocyte-tomonocyte ratio on previously untreated metastatic nonsmall cell lung cancer patients receiving platinum-based doublet. Med Oncol. 2014;31(7):70.

16. Gu XB, Tian T, Tian XJ, Zhang XJ. Prognostic significance of neutrophil-to-lymphocyte ratio in non-small cell lung cancer: a meta-analysis. Sci Rep. 2015;5:12493.

17. Peng B, Wang YH, Liu YM, Ma LX. Prognostic significance of the neutrophil to lymphocyte ratio in patients with non-small cell lung cancer: a systemic review and meta-analysis. Int J Clin Exp Med. 2015;8(3):3098-3106.

18. Yin Y, Wang J, Wang X, Gu L, Pei H, Kuai S, Zhang Y, et al. Prognostic value of the neutrophil to lymphocyte ratio in lung cancer: A meta-analysis. Clinics (Sao Paulo). 2015;70(7):524-530.

19. Hu P, Shen H, Wang G, Zhang P, Liu Q, Du J. Prognostic significance of systemic inflammation-based lymphocyte- monocyte ratio in patients with lung cancer: based on a large cohort study. PLoS One. 2014;9(9):e108062.

20. Nagai Y, Miyazawa H, Huqun, Tanaka T, Udagawa K, Kato M, Fukuyama S, et al. Genetic heterogeneity of the epidermal growth factor receptor in non-small cell lung cancer cell lines revealed by a rapid and sensitive detection system, the peptide nucleic acid-locked nucleic acid PCR clamp. Cancer Res. 2005;65(16):7276-7282.

21. Rami-Porta R, Crowley JJ, Goldstraw P. The revised 
TNM staging system for lung cancer. Ann Thorac Cardiovasc Surg. 2009;15(1):4-9.

22. Minami S, Ogata Y, Ihara S, Yamamoto S, Komuta K. Retrospective analysis of outcomes and prognostic factors of chemotherapy for small-cell lung cancer. Lung Cancer (Auckl). 2016;7:35-44.

23. Minami S, Ogata Y, Ihara S, Yamamoto S, Komuta K. Outcomes and prognostic factors of chemotherapy for patients with locally advanced or metastatic pulmonary squamous cell carcinoma. Lung Cancer (Auckl). 2016;7:99-110.

24. Ando Y, Minami H, Saka H, Ando M, Sakai S, Shimokata K. Adjustment of creatinine clearance improves accuracy of Calvert's formula for carboplatin dosing. Br J Cancer. 1997;76(8):1067-1071.

25. Kanda Y. Investigation of the freely available easy-touse software 'EZR' for medical statistics. Bone Marrow Transplant. 2013;48(3):452-458.

26. Ding PN, Roberts TL, Chua W, Becker TM, Descallar J, Yip PY, Bray V. Clinical outcomes in patients with advanced epidermal growth factor receptor-mutated nonsmall-cell lung cancer in South Western Sydney Local Health District. Intern Med J. 2017;47(12):1405-1411.
27. Lin GN, Peng JW, Liu PP, Liu DY, Xiao JJ, Chen XQ. Elevated neutrophil-to-lymphocyte ratio predicts poor outcome in patients with advanced non-small-cell lung cancer receiving first-line gefitinib or erlotinib treatment. Asia Pac J Clin Oncol. 2017;13(5):e189-e194.

28. Meriggi F, Codignola C, Beretta GD, Ceresoli GL, Caprioli A, Scartozzi M, Fraccon AP, et al. Significance of neutrophil-to-lymphocyte ratio in Western advanced EGFR-mutated non-small cell lung cancer receiving a targeted therapy. Tumori. 2017;103(5):443-448.

29. Sim SH, Beom SH, Ahn YO, Keam B, Kim TM, Lee SH, Kim DW, et al. Pretreatment neutrophil-lymphocyte ratio is not a significant prognostic factor in epidermal growth factor receptor-mutant non-small cell lung cancer patients treated with tyrosine kinase inhibitors. Thorac Cancer. 2016;7(2):161-166.

30. Chen YM, Lai CH, Chang HC, Chao TY, Tseng CC, Fang WF, Wang CC, et al. Baseline and trend of lymphocyte-to-monocyte ratio as prognostic factors in epidermal growth factor receptor mutant non-small cell lung cancer patients treated with first-line epidermal growth factor receptor tyrosine kinase inhibitors. PLoS One. 2015;10(8):e0136252. 